\title{
PCA: Memory Leak Detection using Partial Call-Path Analysis
}

\author{
Wen Li \\ Washington State University, USA \\ li.wen@wsu.edu \\ Yulei Sui \\ The University of Technology Sydney, Australia \\ yulei.sui@uts.edu.au
}

\author{
Haipeng Cai \\ Washington State University, USA \\ haipeng.cai@wsu.edu \\ David Manz \\ Pacific Northwest National Laboratory, USA \\ David.Manz@pnnl.gov
}

\begin{abstract}
Data dependence analysis underlies various applications in software quality assurance, yet existing frameworks/tools for this analysis commonly suffer scalability challenges. We present PCA, a static interprocedural data dependence analyzer for real-world $\mathrm{C}$ programs. PCA performs interprocedural points-to and data-flow analyses with a lightweight design. Most of all, it features a partial call-path (PCA) analysis that consists of optimization options to further speed up data dependence computation. As an example application of it, PCA readily supports memory leak detection, for which it helps achieve close or better performance and precision relative to the same application based on a state-of-the-art value flow analysis. In particular, it found four more memory leaks in an industry-scale system which have been fixed by the developers. Through the data dependence it computes, PCA can enable other applications (e.g., impact analysis and taint analysis).

A demo video for PCA can be found here and tool package here.
\end{abstract}

\section{CCS CONCEPTS}

- Security and privacy $\rightarrow$ Software security engineering; • Theory of computation $\rightarrow$ Program analysis.

\section{KEYWORDS}

LLVM, static analysis, data dependence, efficiency, scalability

\section{ACM Reference Format:}

Wen Li, Haipeng Cai, Yulei Sui, and David Manz. 2020. PCA: Memory Leak Detection using Partial Call-Path Analysis. In Proceedings of the 28th ACM foint European Software Engineering Conference and Symposium on the Foundations of Software Engineering (ESEC/FSE '20), November 8-13, 2020, Virtual Event, USA. ACM, New York, NY, USA, 5 pages. https://doi.org/10. 1145/3368089.3417923

\section{INTRODUCTION}

With their growing size and complexity, modern software systems are increasingly challenging to check against desired properties (e.g., correctness and security). One main approach to this task is to reason about program behaviors with respect to how data are computed and accessed (e.g., in terms of data dependence) in the

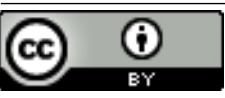

This work is licensed under a Creative Commons Attribution International 4.0 License.

ESEC/FSE '20, November 8-13, 2020, Virtual Event, USA

(C) 2020 Copyright held by the owner/author(s).

ACM ISBN 978-1-4503-7043-1/20/11.

https://doi.org/10.1145/3368089.3417923 program [5, 6]. This approach, as an underlying technique, supports a range of applications in software quality assurance, such as bug detection $[7,19]$ and security vulnerability discovery $[16,17]$.

There are tools that implement this underlying technique or facilitate such implementations. As a recent work, PHASAR [15] provides a generic framework for static interprocedural data flow analysis. Yet rather than a specific tool, it offers building blocks of static analyzers (e.g., call graphs, points-to information) via a set of APIs that tool developers may use to develop their own analyzers (e.g., for computing data dependence). Moreover, based on the IFDS/IDE algorithmic framework [14], PHASAR targets highly precise data flow analyses. For large, complex software systems, efficiency barriers due to its heavyweight nature may not be well paid off by the level of precision it offers-other cost-effectiveness tradeoffs might be more desirable to users [8].

Analyzers of value flow, such as SVF [17] and Pinpoint [16], provide a potential alternative. While a conventional value flow analysis would be built on data dependence analysis hence provide data dependence, these state-of-the-art tools focus on sparse value flow analysis. They focuses on precise computation of value flow information necessary for applications that need it, primarily applicable to source-sink problems such as detecting memory defects. As a result, these frameworks/tools may not provide the best cost-effectiveness tradeoffs for particular dependence-based applications that do not need the information. Commercial tools like CodeSonar [1] and Coverity [2] do so. Yet they are closed-source hence may not be sufficient for research purposes.

Therefore, in this paper, we develop PCA, an open-source static interprocedural data dependence analysis tool that scales to industryscale C software with a practical cost-effectiveness tradeoff that complements to what existing alternatives offer (i..e, primarily focusing on precision or not providing desired balance between analysis cost and effectiveness [15-17]), particularly for solving source-sink problems (e.g., memory leak detection). PCA is built on top of LLVM [13]. It uses the LLVM gold plugin to generate the intermediate representation (IR) code for each module of a given input program and computes interprocedural points-to sets using Andersen's algorithm [4] and LLVM's basic, built-in analysis capabilities. With such information, it then computes data flow facts (e.g., reaching definitions) using a classical fixed-point iterative data-flow analysis algorithm [3]. Finally, it produces the interprocedural data dependence graph. Then in its practical use scenarios, for both practitioners and researchers, PCA enables different (e.g., data dependence based) application/client analyses and tools through its analysis results (e.g., interprocedural control flow and data dependencies). 


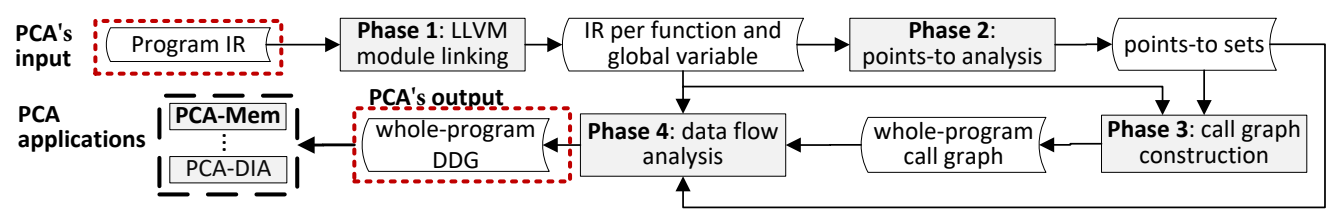

Figure 1: An overview of PCA's architecture, including its input, four phases, output, and applications.

The key merit of PCA is that it offers practical efficiency and scalability for real-world, industry-scale software systems while maintaining a practically useful level of effectiveness in terms of precision. To that end, PCA features a partial call-path analysis (hence our tool name) during the interprocedural data dependence computation, as defined by two levels of performance optimization. First, in the data-flow analysis algorithm, PCA adopts a usercustomizable partial flow sensitivity at function level (i.e., via the call graph) when computing variable definitions and uses while referring to the points-to sets computed by the (Andersen's) flowinsensitive pointer analysis algorithm. This design substantially reduces the overhead of an otherwise heavy (e.g., flow-sensitive) points-to analysis to save the total time cost of PCA while compensating the precision loss from that underlying analysis later (during the dependence computation). Second, PCA opts to ignore constant strings in the points-to analysis to reduce its time and space costs; it further uses integer encoding in storing definitions when performing the data flow analysis to reduce memory use.

To demonstrate the usefulness of PCA, we built PCA-MEM as its example application, a static memory leak detector using the partial call-path analysis. We then evaluate PCA through PCA-MEм by applying it to a standard suite of (small) benchmarks (SPEC2000 $\mathrm{C}$ programs) and an industry-scale, high-performance computing system Slurm [20]. We assessed its efficiency and effectiveness in terms of memory leaks found in comparison to SABER [18], the same application but of the state-of-the-art value flow analysis SVF [17]. On the small SPEC2000 benchmarks, PCA-MEM was close to SABER in both metrics, while on the large system Slurm, PCA-Мем performed better by finding more memory leaks at lower time and space costs. PCA can support more applications than analyzing memory defects through the dependence information it offers.

\section{ARCHITECTURE}

PCA's architecture is depicted in Figure 1. As PCA's input, the per-module IR files of the given program are generated using the LLVM gold plugin [17]. Optionally, users may provide a function blacklist to specify the functions (e.g., those for debugging purposes only) to be skipped by the analysis for better efficiency.

With these user inputs, PCA performs its data flow analysis with optimizations in four phases. In the first phase (LLVM module linking), PCA links the per-module IR files together and parses the functions and global variables of the program, and returns the separated IR for each function and global variable to be used by other phases. The IR code is the output of the LLVM C frontend (Clang) applied against the program, where the gold plugin allows for generating cross-compilation-unit IR code. By preparing the cross-unit IR, this phase is key for the analyses in PCA to be interprocedural. In the second phase (points-to analysis), PCA performs an interprocedural points-to analysis for global, heap,

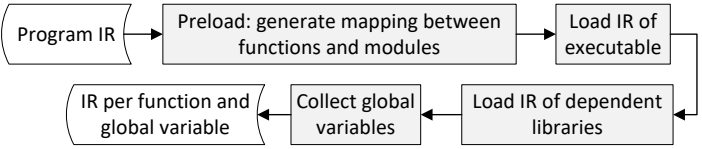

Figure 2: Module linking workflow of PCA.

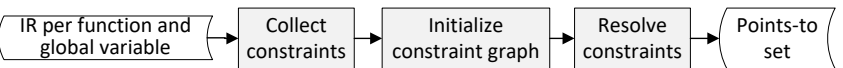

Figure 3: Points-to analysis workflow of PCA.

and local variables, using the Andersen's algorithm [4]. During the third phase (call graph construction), PCA resolves call targets for direct calls with explicit callees and for indirect calls according to the points-to sets returned by the points-to analysis phase. In the last (fourth) phase (data flow analysis), PCA first constructs the interprocedural control flow graph (ICFG) from the given call graph and the LLVM instructions of each function, and then computes data dependence based on the ICFG and the points-to sets. This last phase produces the interprocedural data-dependence graph (DDG) as the final PCA's output. Based on this output, various PCA applications (e.g., taint checking, testing) can be developed. Currently, our tool package includes two applications: PCA-MeM, a static memory leak detector; and PCA-DIA, a dynamic impact analysis tool. For demo purposes, this paper only elaborates PCA-MEM.

\section{LLVM MODULE LINKING}

To enable interprocedural analysis based on LLVM, PCA needs to first link the IR of all the modules of the given input program. Specifically, PCA retrieves all global variables and the definition of all functions in the executable and its dependent libraries, in four major steps as shown in Figure 2 and as elaborated below.

In the first (preload) step, PCA traverses all the (per-module) IR files of the input program, and parses each module to create a mapping between each function defined in the module to the module itself (i.e., its IR). Second, PCA loads the IR of the executable which contains the entry function of the program. It then parses each callsite in this function to check whether there is a callee for which merely the declaration is included in the current module-that is, whether the callee is defined in an external module. If so, PCA retrieves the module (i.e., its path) that defines the function (according to the mapping created before) and inserts the module path into a on-demand list for later loading. Third, PCA loads modules in the on-demand list and identifies functions defined in these modules the same way as functions are discovered in the previous step. To deal with circular library dependencies, PCA ensures each module is loaded once. Finally, PCA collects all global variables defined in the modules that are loaded in the previous two steps, and produces the set of function definitions and global variables (i.e., the IR of each) in the input program. 


\section{POINTS-TO ANALYSIS}

In this phase, PCA takes the IR of each function and global variable from the previous phase to perform interprocedural points-to analysis of the program in three steps as outlined in Figure 3. The alias analysis implementations in LLVM are intraprocedural.

PCA first collects the constraints of stack and heap variables from each function, and also parameters and return values for each call instruction. It models each function in standard C library (libc) as an identity function but allows users to model library function effects differently. Second, from the collected constraints, PCA constructs a constraint graph and initializes a points-to set for each memory object. PCA uses SparseBitVector [10] data structure for efficient set operations. In the next step, PCA adopts the hybrid cycle detection technique [11] for efficient constraint resolution. The technique utilizes both an offline pre-analysis and online detection of strongly connected components to achieve high efficiency. In all, we adopted the fast and imprecise Andersen's algorithm [4] for a flow- and context-insensitive points-to analysis to trade precision in the resulting points-to sets for the efficiency of computing them.

Optimization. To further reduce its overheads, PCA has the default option of ignoring constant strings in this phase. Including these constants would in general significantly increase the size of the resulting points-to sets hence slow down the analysis due to their prevalence in C programs. For example, these constants account for over $80 \%$ of all global variables in Slurm.

\section{CONTROL AND DATA FLOW ANALYSIS}

With the results from the previous phases, PCA now constructs the whole-program call graph, identifying all possible callees according to the points-to information. It performs function-level reachability analysis through a (BFS) traversal of the initial call graph and prunes nodes unreachable from the program entry.

From the resulting call graph and per-function IR, PCA then builds the (intraprocedural) control flow graph (CFG) for each function hence the interprocedural CFG (ICFG) for the entire program as in [12]. To construct the interprocedural data dependence graph (DDG), PCA collects both definite and possible (induced by pointer aliasing) definitions/uses of variables at each instruction of every reachable function. Then, it follows the classical fixed-point iterative data flow analysis algorithm [3] to compute the reaching definitions and build intraprocedural DDGs. Finally, based on the ICFG, PCA computes interprocedural data dependence by adding transitive edges (e.g., actual/formal parameters linkage) among intraprocedural DDGs as in the classical interprocedural slicing [12]

\begin{tabular}{|c|c|c|c|c|}
\hline void FA(int ** & void $\mathbf{F B}($ int $* \mathrm{Fb})\{$ & int main ()\{ & ${ }^{*} \mathrm{Fb}=1$ & \multirow{3}{*}{$\rightarrow \begin{array}{c}* \mathrm{Fb}=1 \\
\mathrm{Fb} \rightarrow(\mathrm{S}, \mathrm{b})\end{array}$} \\
\hline $\begin{array}{l}\text { int } \mathrm{a}=0 \\
{ }^{*} \mathrm{Fa}=\& \mathrm{a}\end{array}$ & $\begin{array}{l}\text { int } b=0 \\
\underline{F b} \equiv \underline{\&} b_{\dot{q}}\end{array}$ & $\begin{array}{l}\text { int } S=0 \\
F B(\& S)\end{array}$ & $\mathrm{Fb}->(S, b, a)$ & \\
\hline \} & $\begin{array}{l}{ }^{*} \mathrm{Fb}=1 ; 1 \\
\mathrm{FA}(\overline{\mathrm{A}} \mathrm{F} \overline{\mathrm{b}})\end{array}$ & \}$^{\text {return 0; }}$ & $\begin{array}{l}\text { Call path: } \\
\text { main }>\text { FB }\end{array}$ & \\
\hline
\end{tabular}

Figure 4: Illustrating part of the partial call-path analysis.

Optimizations. PCA improves its efficiency and scalability in the data flow analysis here through two optimizations, as the main part of our partial call-path analysis.

The first is to reduce the effects of false positives in the points-to sets by considering function-level control-flow reachability (i.e., on the call graph) to a certain depth. The partial flow sensitivity compensates the precision lost in the flow-insensitive points-to analysis without incurring much cost.

To illustrate the idea, consider the example in Figure 4. When identifying possible definitions at $\star \mathrm{Fb}=1$, based on the pointsto results, the definitions of $\{S, b, a\}$ will be identified. However, considering control-flow reachability with respect to call paths of depth 2 (e.g., main- >FB), it is clear that the definition of $a$ should not be included in the set of possible definitions at $\star \mathrm{Fb}=1$. To prune such false definitions, PCA collects the set of definitions (including those in stack, heap, and global data area) along the call paths considered; it then takes the intersection of this set and the original points-to set at the target node (e.g., the node for Fb in this example), resulting in the reduced set of possible definitions (e.g., $\{S, b\}$ in this case). With this reduced set, the following iterative data flow analysis can converge faster hence be accelerated while producing more precise dependence analysis results. PCA allows users to customize this call-path depth threshold as an option based on program complexity. In essence, the optimization safely prunes spurious aliasing-induced data dependence according to control flow up to the given depth on the call graph. Thus, this optimization does not affect the soundness of our analysis.

The second is to use integer encoding in storing data flow facts (variable definitions in particular) to reduce memory usage. As per the algorithm we use, each definition would be conventionally represented as a 2-tuple (instruction, variable), which would take 16 bytes (on a 64bit machine). And four sets of definitions (IN, OUT, GEN, KILL) for each ICFG node need to be maintained during the iterative analysis until the fixed point is reached. For one of the functions in Slurm, for example, the total number of definitions maintained is $17,097,929$, thus computing the reaching definitions in this function alone would consume at least $256 \mathrm{MB}$ memory. PCA encodes definitions with a simple (short) integer index which only takes 4 bytes per definition. This reduces $75 \%$ of the peak memory usage of the data flow analysis-for the same example, only about $64 \mathrm{MB}$ memory is needed.

\section{EXAMPLE APPLICATION: PCA-MEM}

We developed PCA-MEM based on PCA to demonstrate its use (for statically detecting memory leaks). Using the interprocedural DDG from PCA, PCA-MEM addresses this use case as a source-sink problem, with each allocation site considered a source and each free site as a sink. The idea is to check reachability from a source to each corresponding sink against data and control flow conditions.

First, PCA-MEM collects the set $(N)$ of nodes reachable from the source on the DDG through any realizable path by considering different calling contexts leading to each call. Let $S$ be the set of sinks in $\mathrm{N}$-the memory allocated at the source can have more than one free site. If $S$ is empty, then the memory is never freed.

If $S$ is not empty, PCA-MEM will employ both control flow and data dependence information for detecting partial memory leaks along some program paths. A heap object $o$ allocated at a memory allocation site $\ell$ is treated as deallocated if (1) there is a free site (sink) $\ell^{\prime} \in S$ that is control-flow reachable from $\ell$ along the ICFG, and (2) the object freed at $\ell^{\prime}$ must be object $o$ or its alias as determined by our reaching definition analysis. Otherwise, $o$ is treated as a leaked object. PCA-MEм reports both never freed cases and partial leaks. 
Table 1: Memory leak detection results of PCA-MEM versus SABER on ten SPEC2000 benchmarks.

\begin{tabular}{|c|c|c|c|c|c|c|c|c|c|c|}
\hline \multirow{2}{*}{ Program } & \multirow{2}{*}{ Size (KLOC) } & \multirow{2}{*}{ \#Functions } & \multicolumn{4}{|c|}{ PCA-МЕM } & \multicolumn{4}{|c|}{ SABER } \\
\hline & & & Time (seconds) & Peak Memory (GB) & \#Leaks (\#GV) & \#False Alarms & Time (seconds) & Peak Memory (GB) & \#Leaks & \#False Alarms \\
\hline art & 1.2 & 29 & 0.5 & 0.04 & $1(9)$ & 0 & 0.2 & 0.05 & 2 & 1 \\
\hline bzip2 & 4.7 & 77 & 1.5 & 0.07 & 1(9) & 0 & 0.5 & 0.09 & 1 & 0 \\
\hline crafty & 21.2 & 112 & 15 & 0.35 & $0(12)$ & 0 & 2.3 & 0.6 & 0 & 0 \\
\hline equake & 1.5 & 30 & 3 & 0.16 & $0(29)$ & 0 & 0.5 & 0.07 & 0 & 0 \\
\hline gzip & 8.6 & 113 & 1 & 0.05 & $3(0)$ & 1 & 0.6 & 0.1 & 3 & 1 \\
\hline $\mathrm{mcf}$ & 2.5 & 29 & 0.3 & 0.03 & $0(3)$ & 0 & 0.3 & 0.05 & 0 & 0 \\
\hline parser & 11.4 & 327 & 10.2 & 0.2 & $0(10)$ & 0 & 3 & 0.3 & 0 & 0 \\
\hline twolf & 20.5 & 194 & 70 & 0.8 & $3(46)$ & 1 & 7 & 0.7 & 3 & 1 \\
\hline vpr & 17.8 & 275 & 6.6 & 0.16 & 1 (19) & 0 & 2.6 & 0.38 & 1 & 0 \\
\hline mesa & 61.3 & 1109 & 5.9 & 0.3 & $12(4)$ & 7 & 108 & 4.1 & 10 & 5 \\
\hline total & $\begin{array}{l}150.7 \\
\end{array}$ & 2295 & 1114 & 2.16 & $21(141)$ & 9 & 125 & 6.44 & 20 & 8 \\
\hline
\end{tabular}

\section{EVALUATION}

We evaluate PCA via PCA-MEM against SVF's SABER as the baseline, using 10 SPEC2000 C programs and Slurm (v15.8.7) [20], a real-world, industry-scale workload manager that includes 21 subsystems (with sizes ranging from $186 \mathrm{KLOC}$ to $257 \mathrm{KLOC}$ ). We then manually checked and compared all the detected memory leaks, including never-free and partial leaks. Our experiments ran on an Ubuntu 16.04 server with 2.40G CPU and 512GB DDR3.

\subsection{Efficiency and Effectiveness}

Table 1 summarizes the accuracy and time/memory costs of both tools against the SPEC2000 benchmarks. PCA-MEM found 161 leaks with 9 false alarms in about 114 seconds, consuming 2.16 GB memory. In comparison, SABER found 20 leaks with 8 false alarms in about 125 seconds, consuming 6.44 GB memory-the true positives it found were all part of those found by PCA-MEM. As an implementation merit here, PCA-МЕм handles global variables and it thus found associated memory leaks (noted as "\#GV" in the parentheses). SABER dismisses the GV cases [17]. Excluding such cases, PCA-MEM appeared to be very close to the baseline in effectiveness (in terms of the number of leaks reported and that of false positives)-there were no false alarms among the GV cases.

For almost all of the benchmarks, PCA-MEM took longer time than SABER. This was expected and can be explained by the fact that the underlying analysis of SABER provides information (i.e., sparse value flow) that is lesser and cheaper to compute as in [9], compared to PCA (which underlies PCA-MEM). As mentioned earlier, PCA chooses this relatively heavier analysis to compute information for broader applications. The only exception was with mesa, for which SABER was much slower, mainly because its interprocedural analysis is context-sensitive and the value flow computation is fully flow-sensitive. This design incurs especially high costs when there are a large number of functions that form a very deep call graph, which is the case with mesa. In contrast, the partial flow sensitivity of PCA-MEM's underlying analysis saved greatly in such cases. As a result, the total costs were close between the two tools.

For the largest benchmark Slurm (v15.8.7), PCA-MEM reported 21 leaks including $10 \mathrm{GV}$ cases. Four of these have been confirmed and fixed by the developers (in v18.8.7): never freed memory allocated at hostlist.c:3802 and that at hostlist.c:3803; partial leaks of memory allocated at hostlist.c:1328 (leak branch: hostlist.c:2253), and that at hostlist.c:1335 (leak branch: hostlist.c:2253). SABER did not find any of these four-the 10 leaks it reported were all false alarms.

Figure 5 and Figure 6 compare the tools on peak memory usage and time cost ( $y$ axes), respectively, for the 21 Slurm subsystems ( $x$

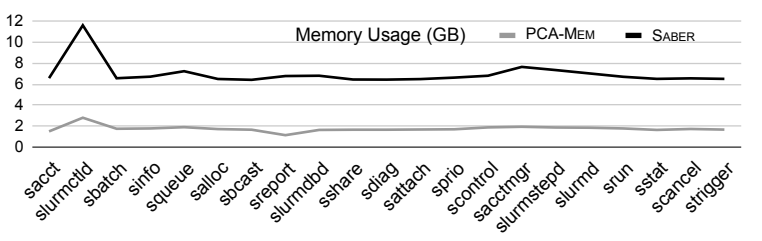

Figure 5: Comparison on peak memory usage against Slurm.

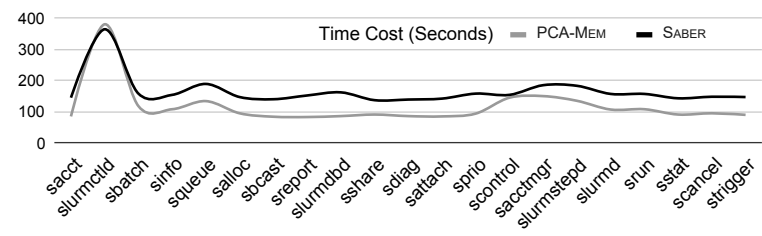

Figure 6: Comparison on time costs against Slurm.

axes). PCA-MEM finished analyzing the entire system (4,079KLOC) in 40 mins with peak memory usage of $2.8 \mathrm{~GB}$, while SABER finished in $58 \mathrm{mins}$ with peak memory usage of $11.6 \mathrm{~GB}$. The reason was similar to that for the mesa case in the SPEC2000 benchmarks. As for those benchmarks, PCA-MEM had higher memory efficiency because of its integer encoding of variable definitions.

\subsection{Limitations}

We made design decisions that lead to imprecision (flow-, field-, and context-insensitivity in computing and using points-to sets) to trade for scalability and efficiency. Thus, the data dependence computed by PCA suffers imprecision. Accordingly, PCA-MEM can give false alarms (as our evaluation results confirmed). Like peer tools (e.g., PHASAR [15]), PCA as a static analyzer does not handle dynamically loaded code.

\section{CONCLUSION}

We presented PCA, a static interprocedural data-flow analyzer for $\mathrm{C}$ programs that offers several efficiency optimization options and different cost-precision tradeoffs from peer tools. Based on PCA we further developed PCA-MEM, a static memory leak detector as an example application of our tool. Through PCA-MEM, we empirically demonstrated PCA's merits in efficiency with practical effectiveness against both standard benchmarks and an industry-scale real-world system. PCA and PCA-MEM are open source and publicly available.

\section{ACKNOWLEDGMENTS}

This work was supported by DOE through PNNL (No. 379101). 


\section{REFERENCES}

[1] August 2018. CodeSonar. https://www.grammatech.com/products/codesonar.

[2] August 2018. Coverity. https://scan.coverity.com/.

[3] Alfred V Aho, Ravi Sethi, and Jeffrey D Ullman. 1986. Compilers, principles, techniques. Addison wesley 7, 8 (1986), 9.

[4] Lars Ole Andersen. 1994. Program Analysis and Specialization for the C Programming Language. (1994).

[5] Haipeng Cai. 2018. Hybrid Program Dependence Approximation for Effective Dynamic Impact Prediction. IEEE Transactions on Software Engineering (TSE) 44 4 (2018), 334-364.

[6] Haipeng Cai and Raul Santelices. 2015. Abstracting Program Dependencies using the Method Dependence Graph. In International Conference on Software Quality, Reliability, and Security (QRS). 49-58.

[7] Haipeng Cai, Raul Santelices, and Siyuan Jiang. 2016. Prioritizing Change Impacts via Semantic Dependence Quantification. IEEE Transactions on Reliability 65, 3 (2016), 1114-1132.

[8] Haipeng Cai, Raul Santelices, and Douglas Thain. 2016. DiaPro: Unifying Dynamic Impact Analyses for Improved and Variable Cost-Effectiveness. ACM Transactions on Software Engineering and Methodology (TOSEM) 25, 2 (2016), 18:1-18:50.

[9] Gang Fan, Rongxin Wu, Qingkai Shi, Xiao Xiao, Jinguo Zhou, and Charles Zhang. 2019. Smoke: scalable path-sensitive memory leak detection for millions of lines of code. In 2019 IEEE/ACM 41st International Conference on Software Engineering (ICSE). IEEE, 72-82.

[10] Ben Hardekopf and Calvin Lin. [n.d.]. Flow-sensitive pointer analysis for millions of lines of code. In $C G O$.

[11] Ben Hardekopf and Calvin Lin. 2007. The ant and the grasshopper: fast and accurate pointer analysis for millions of lines of code. In ACM SIGPLAN Notices,
Vol. 42. ACM, 290-299.

[12] Susan Horwitz, Thomas Reps, and David Binkley. 1990. Interprocedural slicing using dependence graphs. ACM Transactions on Programming Languages and Systems (TOPLAS) 12, 1 (1990), 26-60.

[13] Chris Lattner and Vikram Adve. [n.d.]. LLVM: A compilation framework for lifelong program analysis \& transformation. In CGO.

[14] Mooly Sagiv, Thomas Reps, and Susan Horwitz. 1996. Precise interprocedural dataflow analysis with applications to constant propagation. Theoretical Computer Science 167, 1-2 (1996), 131-170.

[15] Philipp Dominik Schubert, Ben Hermann, and Eric Bodden. 2019. Phasar: An inter-procedural static analysis framework for $\mathrm{c} / \mathrm{c}++$. In International Conference on Tools and Algorithms for the Construction and Analysis of Systems. Springer, 393-410.

[16] Qingkai Shi, Xiao Xiao, Rongxin Wu, Jinguo Zhou, Gang Fan, and Charles Zhang. 2018. Pinpoint: Fast and precise sparse value flow analysis for million lines of code. In ACM SIGPLAN Notices, Vol. 53. ACM, 693-706.

[17] Yulei Sui and Jingling Xue. 2016. SVF: interprocedural static value-flow analysis in LLVM. In Proceedings of the 25th international conference on compiler construction. ACM, 265-266.

[18] Yulei Sui, Ding Ye, and Jingling Xue. 2014. Detecting memory leaks statically with full-sparse value-flow analysis. IEEE Transactions on Software Engineering 40, 2 (2014), 107-122.

[19] Yichen Xie and Alex Aiken. 2005. Saturn: A SAT-based tool for bug detection. In International Conference on Computer Aided Verification. Springer, 139-143.

[20] Andy B Yoo, Morris A Jette, and Mark Grondona. 2003. Slurm: Simple linux utility for resource management. In Workshop on fob Scheduling Strategies for Parallel Processing. Springer, 44-60. 